\title{
COMMON POISSON SHOCK MODELS: APPLICATIONS TO INSURANCE AND CREDIT RISK MODELLING
}

\author{
BY
}

\author{
FiliP LindsKog* AND AleXANDER J. MCNeIL*
}

\begin{abstract}
The idea of using common Poisson shock processes to model dependent event frequencies is well known in the reliability literature. In this paper we examine these models in the context of insurance loss modelling and credit risk modelling. To do this we set up a very general common shock framework for losses of a number of different types that allows for both dependence in loss frequencies across types and dependence in loss severities. Our aims are threefold: to demonstrate that the common shock model is a very natural way of approaching the modelling of dependent losses in an insurance or risk management context; to provide a summary of some analytical results concerning the nature of the dependence implied by the common shock specification; to examine the aggregate loss distribution that results from the model and its sensitivity to the specification of the model parameters.
\end{abstract}

\section{INTRODUCTION}

Suppose we are interested in losses of several different types and in the numbers of these losses that may occur over a given time horizon. More concretely, we might be interested in insurance losses occurring in several different lines of business or several different countries. In credit risk modelling we might be interested in losses related to the default of various types of counterparty. Further suppose that there are strong a priori reasons for believing that the frequencies of losses of different types are dependent. A natural approach to modelling this dependence is to assume that all losses can be related to a series of underlying and independent shock processes. In insurance these shocks might be natural catastrophes; in credit risk modelling they might be a variety of economic events such as local or global recessions; in operational risk modelling they might be the failure of various IT systems. When a shock occurs this may cause losses of several different types; the common shock causes the numbers of losses of each type to be dependent.

\footnotetext{
* Research of the first author was supported by Credit Suisse Group, Swiss Re and UBS AG through RiskLab, Switzerland. We thank in particular Nicole Bäuerle for commenting on an earlier version of this paper.
} 
This kind of construction is very familiar in the reliability literature where the failure of different kinds of system components is modelled as being contingent on independent shocks that may affect one or more components. It is commonly assumed that the different varieties of shocks arrive as independent Poisson processes, in which case the counting processes for the different loss types are also Poisson and can be easily handled analytically. In reliability such models are known as fatal shock models, when the shock always destroys the component, and non-fatal shock models, or not-necessarily-fatal shock models, when components have a chance of surviving the shock. A good basic reference on such models is Barlow and Proschan (1975) and the ideas go back to Marshall and Olkin (1967).

In this paper we set up a very general Poisson shock model; the dimension is arbitrary and shocks may be fatal or not-necessarily-fatal. We review and generalise results for the multivariate Poisson process counting numbers of failures of different types. We also consider the modelling of dependent severities. When a loss occurs, whether in insurance or credit risk modelling, a loss size may be assigned to it. It is often natural to assume that losses of different types caused by the same underlying shock also have dependent severities. We set up general multivariate compound Poisson processes to model the losses of each type. Our interest focusses on three distributions in particular, and their sensitivity to the finer details of the parameterization of the model:

- The multivariate compound Poisson distribution of the cumulative losses of different types at some fixed point in time.

- The multivariate exponential distribution of the times to the first losses of each type.

- The univariate compound Poisson aggregate loss distribution at a fixed time point.

There have been a number of other related papers in this area in recent years, particularly concentrating on the second of these issues. In Savits (1988) nonhomogeneous Poisson shock processes are investigated and the effect of different mean functions for the shock processes on the distributional properties of the joint component lifetimes is studied. In $\mathrm{Li}$ and $\mathrm{Xu}$ (2001) the authors investigate stochastic bounds and dependence properties of the joint component lifetime distribution for rather general shock arrival processes. In particular the effect of dependent interarrival times of the shocks and the effect of simultaneous shock arrivals on the joint component lifetime distribution are investigated; the joint impact of these two types of dependency on the behaviour of the system is analysed.

The present paper is structured as follows. In Section 2 we describe the general not-necessarily-fatal-shock model with dependent loss frequencies and dependent loss severities. In Section 3 we ignore loss severities and examine the multivariate distribution of loss frequencies and the consequences for the aggregate loss frequency distribution of specifying the shock structure in different ways. An important key to analysing the model is to see that it may be written in terms of an equivalent fatal shock model. This facilitates the approximation of the aggregate loss frequency distribution using the Panjer recursion approach 
and also makes it very easy to analyse the multivariate exponential distribution of the times to the first losses of each type. In section 4 the analysis is generalised by including dependent loss severities. The dependence in severities is created using copula techniques and the object of interest is now the tail of the overall aggregate loss distribution. Sections 3 and 4 are illustrated with a stylized insurance example; Section 5 consists of an extended example of how the model might be applied to the modelling of portfolio credit risk.

\section{THE Model}

\subsection{Loss Frequencies}

Suppose there are $m$ different types of shock or event and, for $e=1, \ldots, m$, let

$$
\left\{N^{(e)}(t), t \geq 0\right\}
$$

be a Poisson process with intensity $\lambda^{(e)}$ recording the number of events of type $e$ occurring in $(0, t]$. Assume further that these shock counting processes are independent. Consider losses of $n$ different types and, for $j=1, \ldots, n$, let

$$
\left\{N_{j}(t), t \geq 0\right\}
$$

be a counting process that records the frequency of losses of the $j$ th type occurring in $(0, t]$.

At the $r$ th occurrence of an event of type $e$ the Bernoulli variable $I_{j, r}^{(e)}$ indicates whether a loss of type $j$ occurs. The vectors

$$
\mathbf{I}_{r}^{(e)}=\left(I_{1, r}^{(e)}, \ldots, I_{n, r}^{(e)}\right)^{\prime}
$$

for $r=1, \ldots, N^{(e)}(t)$ are considered to be independent and identically distributed with a multivariate Bernoulli distribution. In other words, each new event represents a new independent opportunity to incur a loss but, for a fixed event, the loss trigger variables for losses of different types may be dependent. The form of the dependence depends on the specification of the multivariate Bernoulli distribution and independence is a special case. We use the following notation for $p$-dimensional marginal probabilities of this distribution (where the subscript $r$ is dropped for simplicity).

$$
P\left(I_{j_{1}}^{(e)}=i_{j_{1}}, \ldots, I_{j_{p}}^{(e)}=i_{j_{p}}\right)=p_{j_{1}, \ldots, j_{p}}^{(e)}\left(i_{j_{1}}, \ldots, i_{j_{p}}\right), i_{j_{1}}, \ldots, i_{j_{p}} \in\{0,1\} .
$$

We also write $p_{j}^{(e)}(1)=p_{j}^{(e)}$ for one-dimensional marginal probabilities, so that in the special case of conditional independence we have

$$
p_{j_{1}, \ldots, j_{p}}^{(e)}(1, \ldots, 1)=\prod_{k=1}^{p} p_{j_{k}}^{(e)}
$$


The counting processes for events and losses are thus linked by

$$
N_{j}(t)=\sum_{e=1}^{m} \sum_{r=1}^{N^{(e)}(t)} I_{j, r}^{(e)} .
$$

Under the Poisson assumption for the event processes and the Bernoulli assumption for the loss indicators, the loss processes $\left\{N_{j}(t), t \geq 0\right\}$ are clearly Poisson themselves, since they are obtained by superpositioning $m$ independent (possibly thinned) Poisson processes generated by the $m$ underlying event processes. $\left(N_{1}(t), \ldots, N_{n}(t)\right)^{\prime}$ can be thought of as having a multivariate Poisson distribution.

However the total number of losses $N(t)=\sum_{j=1}^{n} N_{j}(t)$ is in general not Poisson but rather compound Poisson. It is the sum of $m$ independent compound Poisson distributed random variables as can be seen by writing

$$
N(t)=\sum_{e=1}^{m} \sum_{r=1}^{N^{(e)}(t)} \sum_{j=1}^{n} I_{j, r}^{(e)}
$$

The compounding distribution of the eth compound Poisson process is the distribution of $\sum_{j=1}^{n} I_{j}^{(e)}$, which in general is a sum of dependent Bernoulli variables. We return to the compound Poisson nature of the process $\{N(t), t \geq 0\}$ after generalising it in the next section.

\subsection{Adding Dependent Severities}

We can easily add severities to our multivariate Poisson model. Suppose that when the $r$ th event of type $e$ occurs a potential loss of type $j$ with severity $X_{j, r}^{(e)}$ can occur. Whether the loss occurs or not is of course determined by the value of the indicator $I_{j, r}^{(e)}$, which we assume is independent of $X_{j, r}^{(e)}$. The potential losses $\left\{X_{j, r}^{(e)}, r=1, \ldots, N^{(e)}(t), e=1, \ldots, m\right\}$ are considered to be iid with distribution $F_{j}$. Potential losses of different types caused by the same event may however be dependent. We consider that they have a joint distribution function $F$. That is, for a vector $\mathbf{X}_{r}^{(e)}$ of potential losses generated by the same event we assume

$$
\mathbf{X}_{r}^{(e)}=\left(X_{1, r}^{(e)}, \ldots, X_{n, r}^{(e)}\right)^{\prime} \sim F .
$$

In a more general model it would be possible to make the multivariate distribution of losses caused by the same event depend on the nature of the underlying event $e$. However, in practice it may make sense to assume that there is a single underlying multivariate severity distribution which generates the severities for all event types. This reflects the fact that it is often standard practice in insurance to model losses of the same type type as having an identical claim size distribution, without necessarily differentiating carefully between the events that caused them. 
The aggregate loss process for losses of type $j$ is a compound Poisson process given by

$$
Z_{j}(t)=\sum_{e=1}^{m} \sum_{r=1}^{N^{(e)}(t)} I_{j, r}^{(e)} X_{j, r}^{(e)}
$$

The aggregate loss caused by losses of all types can be written as

$$
Z(t)=\sum_{e=1}^{m} \sum_{r=1}^{N^{(e)}(t)} \sum_{j=1}^{n} I_{j, r}^{(e)} X_{j, r}^{(e)}=\sum_{e=1}^{m} \sum_{r=1}^{N^{(e)}(t)} \mathbf{I}_{r}^{(e)^{\prime}} \mathbf{X}_{r}^{(e)},
$$

and is again seen to be a sum of $m$ independent compound Poisson distributed random variables, and therefore itself compound Poisson distributed. Clearly (2) is a special case of (4) and (1) is a special case of (3). Thus we can understand all of these processes by focusing on (4). The compound Poisson nature of $Z(t)$ can be clarified by rewriting this process as

$$
Z(t) \stackrel{\mathrm{d}}{=} \sum_{s=1}^{S(t)} Y_{s}
$$

where $\{S(t), t \geq 0\}$ is a Poisson process with intensity $\lambda=\sum_{e=1}^{m} \lambda^{(e)}$, counting all shocks $s$ generated by all event types, and where the random variables $Y_{1}, \ldots, Y_{S(t)}$ are iid and independent of $\{S(t), t \geq 0\} . Y_{1}$ has the stochastic representation

$$
\left.Y_{1} \stackrel{\mathrm{d}}{=}\left(\sum_{e=1}^{m} 1_{\left(\sum_{j=1}^{e-1} \lambda()\right) \lambda, \Sigma_{j=1}^{e}\left(\lambda^{(j)}\right)}\right)(U) \mathbf{I}^{(e)}\right)^{\prime} \mathbf{X}
$$

where $U, \mathbf{I}^{(e)}, \mathbf{X}$ are independent, $U$ is uniformly distributed on $(0,1), \mathbf{I}^{(e)}$ is a generic random vector of indicators for shocks of event type $e$, and $\mathbf{X}$ is a generic random vector of severities caused by the same shock. In words: a shock $s$ is of event type $e$ with probability $\lambda^{(e)} / \lambda$.

We consider two examples that fit into the framework of the model we have set up. The first one, an insurance application of the model, we continue to develop throughout the paper. The second one, a credit risk application, is presented separately in Section 5.

\subsection{Insurance example: natural catastrophe modelling}

Fix $n=2, m=3$. Let $N_{1}(t)$ and $N_{2}(t)$ count windstorm losses in France and Germany respectively. Suppose these are generated by three different kinds of windstorm that occur independently. $N^{(1)}(t)$ counts west European windstorms; these are likely to cause French losses but no German losses. $N^{(2)}(t)$ counts central European windstorms; these are likely to cause German losses but no French losses. $N^{(3)}(t)$ counts pan-European windstorms, which are likely to cause both French and German losses. 


\section{The Effect of Dependent Loss Frequencies}

To begin with we look at the distribution of the random vector $\left(N_{1}(t), \ldots\right.$, $\left.N_{n}(t)\right)^{\prime}$, particularly with regard to its univariate and bivariate margins as well as the correlation structure. Part 2 of the following proposition is from Barlow and Proschan (1975), p. 137.

\section{Proposition 1.}

1. $\left\{\left(N_{1}(t), \ldots, N_{n}(t)\right)^{\prime}, t \geq 0\right\}$ is a multivariate Poisson process with

$$
E\left(N_{j}(t)\right)=t \sum_{e=1}^{m} \lambda^{(e)} p_{j}^{(e)} .
$$

2. The two-dimensional marginals are given by

$$
\begin{aligned}
& P\left(N_{j}(t)=n_{j}, N_{k}(t)=n_{k}\right)=e^{-\lambda t\left(p_{j, k}(1,1)+p_{j, k}(1,0)+p_{j, k}(0,1)\right) \times} \\
& \sum_{i=0}^{\min \left\{n_{j}, n_{k}\right\}} \frac{\left(\lambda t p_{j, k}(1,1)\right)^{i}\left(\lambda t p_{j, k}(1,0)\right)^{n_{j}-i}\left(\lambda t p_{j, k}(0,1)\right)^{n_{k}-i}}{i !\left(n_{j}-i\right) !\left(n_{k}-i\right) !},
\end{aligned}
$$

where $\lambda=\sum_{e=1}^{m} \lambda^{(e)}$ and

$$
p_{j, k}\left(i_{j}, i_{k}\right)=\lambda^{-1} \sum_{e=1}^{m} \lambda^{(e)} p_{j, k}^{(e)}\left(i_{j}, i_{k}\right), i_{j}, i_{k} \in\{0,1\} .
$$

3. The covariance and correlation structure is given by

$$
\operatorname{cov}\left(N_{j}(t), N_{k}(t)\right)=t \sum_{e=1}^{m} \lambda^{(e)} p_{j, k}^{(e)}(1,1)
$$

and

$$
\rho\left(N_{j}(t), N_{k}(t)\right)=\frac{\sum_{e=1}^{m} \lambda^{(e)} p_{j, k}^{(e)}(1,1)}{\sqrt{\left(\sum_{e=1}^{m} \lambda^{(e)} p_{j}^{(e)}\right)\left(\sum_{e=1}^{m} \lambda^{(e)} p_{k}^{(e)}\right)}} .
$$

\section{Proof}

1. obvious using thinning and superposition arguments for independent Poisson processes.

2. is found in Barlow and Proschan (1975), p. 137.

3. is a special case of Proposition 7 (part 2).

Clearly, from Proposition 1 part 3, a necessary condition for $N_{j}(t)$ and $N_{k}(t)$ to be independent is that $p_{j, k}^{(e)}(1,1)=0$ for all $e$; i.e. it must be impossible for 
losses of types $j$ and $k$ to be caused by the same event. If for at least one event it is possible that both loss types occur, then we have positive correlation between loss numbers. However Proposition 1, part 2 allows us to make a stronger statement.

Corollary 2. $N_{j}(t)$ and $N_{k}(t)$ are independent if and only if $p_{j, k}^{(e)}(1,1)=0$ for all $e$.

Note that if $p_{j, k}^{(e)}(1,1)=0$ for all $j, k$ with $j \neq k$, then

$$
\begin{aligned}
P\left(\mathbf{I}_{r}^{(e)}=\mathbf{0}\right) & =1-P\left(\cup_{j=1}^{n}\left\{I_{j, r}^{(e)}=1\right\}\right) \\
& =1-\left(\sum_{j=1}^{n} p_{j}^{(e)}-\sum_{j<k} p_{j, k}^{(e)}(1,1)+\ldots+(-1)^{n-1} p_{1, \ldots, n}^{(e)}(1, \ldots, 1)\right) \\
& =1-\sum_{j=1}^{n} p_{j}^{(e)} .
\end{aligned}
$$

Hence if $\sum_{j=1}^{n} p_{j}^{(e)}>1$ for some $e$, then $p_{j, k}^{(e)}(1,1)>0$ for some $j \neq k$, or equivalently:

Corollary 3. If $\sum_{j=1}^{n} p_{j}^{(e)}>1$ for some e, then $N_{1}(t), \ldots, N_{n}(t)$ are not independent.

Thus if we begin by specifying univariate conditional loss probabilities $p_{j}^{(e)}$ it is not always true that a shock model can be constructed which gives independent loss frequencies.

We have already noted that the process of total loss numbers $N(t)=$ $\sum_{j=1}^{n} N_{j}(t)$ is in general not Poisson (but rather a sum of independent compound Poissons). If there is positive correlation between components $N_{j}(t)$ then $\{N(t), t \geq 0\}$ itself cannot be a Poisson process since it is overdispersed with respect to Poisson. It can easily be calculated (see Proposition 9 later) that

$$
\operatorname{var}(N(t))=\sum_{j=1}^{n} \sum_{k=1}^{n} \operatorname{cov}\left(N_{j}(t), N_{k}(t)\right)>E(N(t))
$$

Suppose we define a new vector of independent Poisson distributed loss counters $\hat{N}_{j}(t)$ such that $\hat{N}_{j}(t) \stackrel{\text { d }}{=} N_{j}(t)$. Clearly $\hat{N}(t)=\sum_{j=1}^{n} \hat{N}_{j}(t)$ is Poisson distributed and

$$
\operatorname{var}(\hat{N}(t))=E(\hat{N}(t))=E(N(t)) .
$$

The case where the components $N_{j}(t)$ are dependent is clearly more dangerous (in the sense of higher variance) than the case with independent components. Although the expected number of total losses is the same in both cases the 
variance is higher in the dependent case and, using (9) and (8), we can calculate the inflation of the variance that results from dependence.

\subsection{Insurance example (continued)}

Consider a 5 year period and suppose French losses occur on average 5 times per year and German losses on average 6 times per year; in other words we assume $\lambda_{1}=5$ and $\lambda_{2}=6$. We consider three models for the dependence between these loss frequencies.

- Case 1: No common shocks. If there are no common shocks, then $N(5)=$ $N_{1}(5)+N_{2}(5)$ has a Poisson distribution with intensity $\lambda=\lambda_{1}+\lambda_{2}=5+6=$ 11.

In reality we believe that there are common shocks, in our case particularly the pan-European windstorms. Suppose west, central and pan-European windstorms occur on average 4, 3 and 3 times per year respectively. In terms of event intensities we have

$$
\lambda^{(1)}=4, \lambda^{(2)}=3 \text { and } \lambda^{(3)}=3 .
$$

In terms of the indicator probabilities we assume that empirical evidence and expert judgement has been used to estimate

$$
p_{1}^{(1)}=1 / 2, p_{2}^{(1)}=1 / 4, p_{1}^{(2)}=1 / 6, p_{2}^{(2)}=5 / 6, p_{1}^{(3)}=5 / 6 \text { and } p_{2}^{(3)}=5 / 6
$$

which means that, although unlikely, west European windstorms can cause German losses and central European windstorms can cause French losses. Note that these choices provide an example where the assumption of no common shocks is not only unrealistic but also impossible. To see this consider Corollary 3 and note that $p_{1}^{(3)}+p_{2}^{(3)}>1$.

To make sure that our estimates of event frequencies and indicator probabilities tally with our assessment of loss frequencies we must have that

$$
\lambda_{j}=\lambda^{(1)} p_{j}^{(1)}+\lambda^{(2)} p_{j}^{(2)}+\lambda^{(3)} p_{j}^{(3)}, j=1,2 .
$$

However the specification of the univariate indicator probabilities is insufficient to completely specify the model. We need to fix the dependence structure of the bivariate indicators $\left(I_{1}^{(e)}, I_{2}^{(e)}\right)^{\prime}$ for $e=1,2,3$. For simplicity we will consider two possibilities.

\section{- Case 2: Independent indicators.}

$$
p_{1,2}^{(e)}(1,1)=p_{1}^{(e)} p_{2}^{(e)} \text { for } e=1,2,3 .
$$




\section{- Case 3: Positively dependent indicators.}

$$
p_{1,2}^{(e)}(1,1) \geq p_{1}^{(e)} p_{2}^{(e)} \text { for } e=1,2,3 .
$$

To be specific in Case 3 we will consider $p_{1,2}^{(e)}(1,1)=\min \left(p_{1}^{(e)}, p_{2}^{(e)}\right)$, which is the strongest possible dependence between the indicators, sometimes known as comonotonicity; the random variables $X_{1}, \ldots, X_{n}$ are said to be comonotonic if there exist increasing functions $v_{1}, \ldots, v_{n}: \mathbb{R} \rightarrow \mathbb{R}$, and a random variable $Z$ such that $\left(X_{1}, \ldots, X_{n}\right)^{\prime} \stackrel{\mathrm{d}}{=}\left(v_{1}(Z), \ldots, v_{n}(Z)\right)^{\prime}$. For more on comonotonicity see Wang and Dhaene (1998) and the references therein. See also Joe (1997) for some discussion of dependence bounds in multivariate Bernoulli models. In terms of interpretation in our application this means:

- if a west European windstorm causes a German loss, then with certainty it also causes a French loss;

- if a central European windstorm causes a French loss, then with certainty it also causes a German loss;

- if a pan-European windstorm causes one kind of loss, then with certainty it causes the other kind of loss.

For cases 1, 2 and 3 we get $\operatorname{var}(N(5))=55,85$ and 95 respectively. Of more interest than the variance as a measure of the riskiness of $N(5)$ are the tail probabilities $P(N(5)>k)$. In this example these probabilities can be calculated analytically using formula (7) for the bivariate frequency function. The left plot in Figure 1 shows exceedence probabilities $P(N(5)>k)$, for
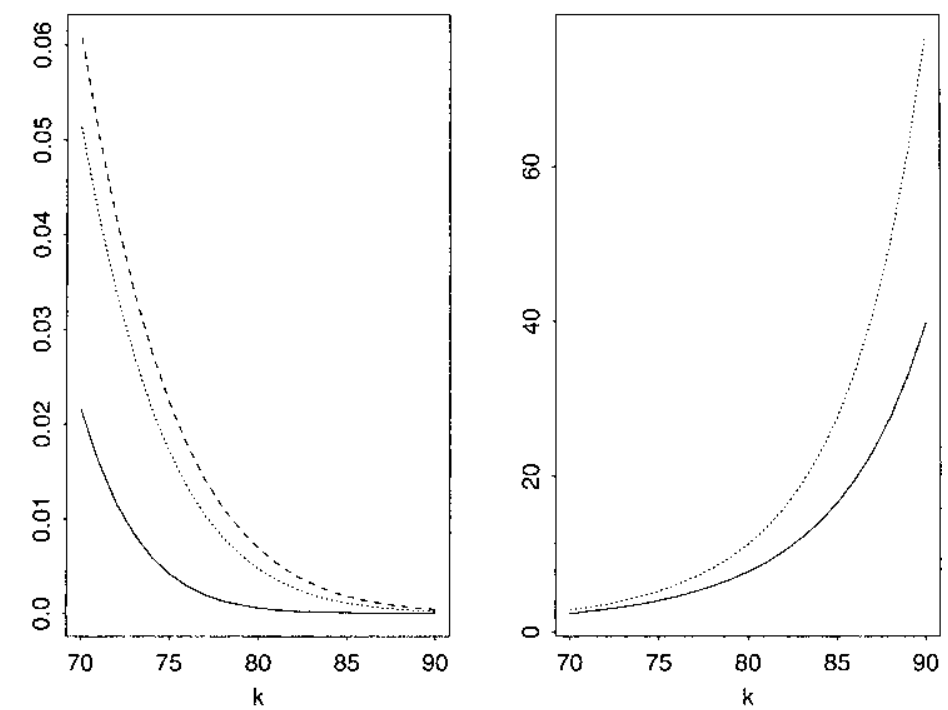

Figure 1: Left: Exceedence probabilities $P(N(5)>k)$ for $k=70,71, \ldots, 90$, for case 1 (lower), 2 (middle) and 3 (upper). Right: Ratios of such exceedence probabilities for cases 1-2 (lower) and 1-3 (upper). 
$k=70,71, \ldots, 90$, for the three cases. The right plot shows by which factor such an exceedence probability is underestimated by case 1 if the correct model would be given by case 2 or 3 . Clearly, both the presence of common shocks and then the subsequent addition of dependent indicators have a profound effect on the aggregate frequency distribution of $N(5)$.

\subsection{The Equivalent Fatal Shock Model}

The not-necessarily-fatal shock model set up in the previous section has the nice property of being easily interpreted. As we will now show this model has an equivalent representation as a fatal shock model. Basically, instead of counting all shocks, we only count loss-causing shocks. From this representation we can draw a number of non-trivial conclusions about our original model.

Let $S$ be the set of non-empty subsets of $\{1, \ldots, n\}$. For $s \in \mathcal{S}$ we introduce a new counting process $\tilde{N}_{s}(t)$, which counts shocks in $(0, t]$ resulting in losses of all types in $s$ only. Thus if $s=\{1,2,3\}$, then $\tilde{N}_{s}(t)$ counts shocks which cause simultaneous losses of types 1, 2 and 3, but not of types 4 to $n$. We have

$$
\tilde{N}_{s}(t)=\sum_{e=1}^{m} \sum_{r=1}^{N^{(e)}(t)} \sum_{s^{\prime}: s^{\prime} \supseteq s}(-1)^{\left|s^{\prime}\right|-|s|} \prod_{k \in s^{\prime}} I_{k, r}^{(e)},
$$

where $\sum_{s^{\prime}: s^{\prime} \supseteq s}(-1)^{\left|s^{\prime}\right|-|s|} \prod_{k \in s^{\prime}} I_{k, r}^{(e)}$ is an indicator random variable which takes the value 1 if the $r$ th shock of type $e$ causes losses of all type in $s$ only, and the value 0 otherwise. Furthermore let $\tilde{N}(t)$ count all shocks in $(0, t]$ which result in losses of any kind. Clearly we have

$$
\tilde{N}(t)=\sum_{s \in S} \tilde{N}_{s}(t)
$$

The key to a fatal shock representation is the following result.

\section{Proposition 4.}

1. $\left\{\tilde{N}_{s}(t), t \geq 0\right\}$ for $s \in \mathcal{S}$ are independent Poisson processes with intensities

$$
\lambda_{s}=\sum_{e=1}^{m} \lambda^{(e)} \sum_{s^{\prime}: s^{\prime} \supseteq s}(-1)^{\left|s^{\prime}\right|-|s|} p_{s^{\prime}}^{(e)},
$$

where $p_{s^{\prime}}^{(e)}=P\left(\prod_{k \in s^{\prime}} I_{k, r}^{(e)}=1\right)$, and

2. $\{\tilde{N}(t), t \geq 0\}$ is a Poisson process with intensity

$$
\tilde{\lambda}=\sum_{s \in \mathcal{S}} \lambda_{s}=\sum_{e=1}^{m} \lambda^{(e)}\left(1-P\left(\mathbf{I}_{r}^{(e)}=\mathbf{0}\right)\right) .
$$


Proof. Let $J_{s, r}^{(e)}=\sum_{s^{\prime} s^{\prime} \supseteq s}(-1)^{\left|s^{\prime}\right|-|s|} \prod_{k \in s^{\prime}} I_{k, r^{\prime}}^{(e)}$. First note that the random variable $J_{s, r}^{(e)}$ takes values in $\{0,1\}$, and that $P\left(J_{s, r}^{(e)}=1\right)=\sum_{s^{\prime} s^{\prime} \supseteq s}(-1)^{\left|s^{\prime}\right|-|s|} p_{s^{\prime}}^{(e)}$, where $p_{s^{\prime}}^{(e)}=P\left(\prod_{k \in s^{\prime}} I_{k, r}^{(e)}=1\right)$, does not depend on $r$. Hence $\left\{\sum_{r=1}^{N^{(e)}(t)} J_{s, r}^{(e)}, t \geq 0\right\}$ is obtained by thinning the Poisson process $\left\{N^{(e)}(t), t \geq 0\right\}$, and is therefore a Poisson process with intensity $\lambda^{(e)} \sum_{s^{\prime}: s^{\prime} \supseteq s}(-1)^{\left|s^{\prime}\right|-|s|} p_{s^{\prime}}^{(e)} \cdot\left\{\tilde{N}_{s}(t), t \geq 0\right\}$ is obtained by superpositioning the independent Poisson processes $\left\{\sum_{r=1}^{N^{(e)}(t)} J_{s, r}^{(e)}, t \geq 0\right\}$ for $e=1, \ldots, m$, and is therefore a Poisson process with intensity $\lambda_{s}=\sum_{e=1}^{m} \lambda^{(e)}$ $\sum_{s^{\prime}: s^{\prime} \supseteq s}(-1)^{\left|s^{\prime}\right|-|s|} p_{s^{\prime}}^{(e)} . \quad$ Since $\left.P\left(\sum_{s \in S} \sum_{s^{\prime}: s^{\prime} \supseteq s}(-1)^{\left|s^{\prime}\right|-|s|} \prod_{k \in s^{\prime}} I_{k, r}^{(e)}=1\right)\right)$ (the probability that the $r$ th shock of type $e$ causes at least one loss) does not depend on $r$, thinning and superpositioning arguments give that $\{\tilde{N}(t), t \geq 0\}$ is a Poisson process with intensity $\lambda=\sum_{s \in s} \lambda_{s}=\sum_{e=1}^{m} \lambda^{(e)}\left(1-P\left(\mathbf{I}_{r}^{(e)}=\mathbf{0}\right)\right)$. Each jump in the process $\{\tilde{N}(t), t \geq 0\}$ corresponds to a jump in exactly one of the processes $\left\{\tilde{N}_{s}(t), t \geq 0\right\}$ for $s \in \mathcal{S}$. Given a jump in $\{\tilde{N}(t), t \geq 0\}$, the probability of the jump being in $\left\{\tilde{N}_{s}(t), t \geq 0\right\}$ is given by $q_{s}=\lambda_{s} / \tilde{\lambda}$ for $s \in \mathcal{S}$. Order the $l=|\mathcal{S}|=2^{n}-1$ non-empty subsets of $\{1, \ldots, n\}$ in some arbitrary way.

Then

$$
P\left(\tilde{N}_{s_{1}}(t)=n_{1}, \ldots, \tilde{N}_{s_{l}}(t)=n_{l} \mid \tilde{N}(t)=\tilde{n}\right)= \begin{cases}\tilde{n} ! \prod_{j=1}^{l}\left(q_{s_{j}}^{n_{j}} / n_{j} !\right), \tilde{n}=\sum_{j=1}^{l} n_{j} \\ 0 \quad, \tilde{n} \neq \sum_{j=1}^{l} n_{j} .\end{cases}
$$

and hence

$$
\begin{aligned}
P\left(\tilde{N}_{s_{1}}(t)=n_{1}, \ldots, \tilde{N}_{s_{l}}(t)=n_{l}\right) & =P\left(\tilde{N}(t)=\sum_{j=1}^{l} n_{j}\right)\left(\sum_{j=1}^{l} n_{j}\right) ! \prod_{j=1}^{l} \frac{q_{s_{j}}^{n_{j}}}{n_{j} !} \\
& =\prod_{j=1}^{l} e^{-\lambda_{s_{j}} t} \frac{\left(\lambda_{s_{j}} t\right)^{n_{j}}}{n_{j} !}=\prod_{j=1}^{l} P\left(\tilde{N}_{s_{j}}(t)=n_{j}\right) .
\end{aligned}
$$

It follows that the processes $\left\{\tilde{N}_{s}(t), t \geq 0\right\}$ for $s \in \mathcal{S}$ are independent Poisson processes.

Since the Poisson processes $\left\{\tilde{N}_{s}(t), t \geq 0\right\}$ for $s \in \mathcal{S}$ are independent and since the loss counting processes may be written as

$$
N_{j}(t)=\sum_{s: j \in s} \tilde{N}_{s}(t),
$$


it also follows that we have obtained a fatal shock model representation for the original not-necessarily-fatal set-up.

Furthermore, since $\lambda_{s}=0$ for all $s$ with $|s| \geq 2$ if and only if $p_{j, k}^{(e)}(1,1)=0$ for all $e$ and all $j, k$ with $j \neq k$, Corollary 2 concerning pairwise independence can be strengthened.

Corollary 5. $N_{1}(t), \ldots, N_{n}(t)$ are independent if and only if $p_{j, k}^{(e)}(1,1)=0$ for all $e$ and all $j, k$ with $j \neq k$.

A direct consequence of the fatal shock model representation of the original not-necessarily-fatal shock model is that the multivariate distribution of the times to first losses can be easily analysed. Let $T_{j}=\inf \left\{t: N_{j}(t)>0\right\}$ denote the time to the first loss of type $j$. We now consider briefly the distribution of $\left(T_{1}, \ldots, T_{n}\right)^{\prime}$ whose dependence structure is well understood. For $s \in \mathcal{S}$ let $Z_{s}=\inf \left\{t: \tilde{N}_{s}(t)>0\right\} .\left\{Z_{s}\right\}_{s \in S}$ are independent exponential random variables with parameters $\left\{\lambda_{s}\right\}_{s \in S}$. Hence

$$
T_{j}=\inf \left\{t: N_{j}(t)>0\right\}=\inf \left\{t: \sum_{s: j \in s} \tilde{N}_{s}(t)>0\right\}=\min _{s: j \in s} Z_{s}
$$

and $\left(T_{1}, \ldots, T_{n}\right)=\left(\min _{s: 1 \in s} Z_{s}, \ldots, \min _{s: n \in s} Z_{s}\right)$. Survival probabilities for $\left(T_{1}, \ldots, T_{n}\right)$ can be calculated as follows.

$$
\begin{aligned}
& P\left(T_{1}>t_{1}, \ldots, T_{n}>t_{n}\right) \\
& =P\left(\cap_{i} \tilde{N}_{\{i\}}\left(t_{i}\right)=0, \cap_{i<j} \tilde{N}_{\{i, j\}}\left(\max \left(t_{i}, t_{j}\right)\right)=0, \ldots, \tilde{N}_{\{1, \ldots, n\}}\left(\max \left(t_{1}, \ldots, t_{n}\right)\right)=0\right) \\
& =\prod_{i} P\left(\tilde{N}_{\{i\}}\left(t_{i}\right)=0\right) \prod_{i<j} P\left(\tilde{N}_{\{i, j\}}\left(\max \left(t_{i}, t_{j}\right)\right)=0\right) \cdots P\left(\tilde{N}_{\{1, \ldots, n\}}\left(\max \left(t_{1}, \ldots, t_{n}\right)\right)=0\right) \\
& =\exp \left(-\sum_{i} \lambda_{\{i\}} t_{i}-\sum_{i<j} \lambda_{\{i, j\}} \max \left(t_{i}, t_{j}\right)-\ldots-\lambda_{\{1, \ldots, n\}} \max \left(t_{1}, \ldots, t_{n}\right)\right)
\end{aligned}
$$

The multivariate exponential distribution with this joint survival probability is the multivariate exponential distribution of Marshall and Olkin (Marshall and Olkin (1967)). The distribution has been studied extensively, see Barlow and Proschan (1975), Joe (1997), Marshall and Olkin (1967) or Nelsen (1999). The multivariate exponential distribution of Marshall and Olkin has the property that

$$
P\left(T_{1}>t_{1}+s_{1}, \ldots, T_{n}>t_{n}+s_{n} \mid T_{1}>t_{1}, \ldots, T_{n}>t_{n}\right)=P\left(T_{1}>s_{1}, \ldots, T_{n}>s_{n}\right),
$$

for all $t_{1}, \ldots, t_{n}, s_{1}, \ldots, s_{n}>0$. This is the multivariate version of the lack of memory property which is well known for the univariate exponential distribution. Not that this does not apply to general multivariate distributions with 
exponential marginals. The expression for the joint survival probability (10) might not be very convenient to work with if the model was set up as a notnecessarily-fatal shock model. However, it can easily be rewritten in a more convenient form.

$$
\begin{aligned}
P\left(T_{1}>t_{1}, \ldots, T_{n}>t_{n}\right)=\exp \left(-\sum_{e=1}^{m} \lambda^{(e)}\left[\sum_{i} p_{i}^{(e)} t_{i}+\sum_{i<j} p_{i, j}^{(e)}(1,1) \min \left(t_{i}, t_{j}\right)\right.\right. \\
\left.\left.+\ldots+p_{1, \ldots, n}^{(e)}(1, \ldots, 1) \min \left(t_{1}, \ldots, t_{n}\right)\right]\right) .
\end{aligned}
$$

Recall that for a Poisson process with intensity $\mu$, the time to the $k$ th jump is $\Gamma(k, 1 / \mu)$-distributed, where $\Gamma(\cdot, \cdot)$ denotes the Gamma distribution. Hence the time to the $k$ th loss-causing shock is $\Gamma(k, 1 / \tilde{\lambda})$-distributed, where $\tilde{\lambda}=\sum_{e=1}^{m} \lambda^{(e)}\left(1-P\left(\mathbf{I}_{r}^{(e)}=\mathbf{0}\right)\right)$. The time to the $k$ th loss is inf $\{t: N(t) \geq k\}$, where

$$
N(t)=\sum_{i=1}^{n} i \sum_{s:|s|=i} \tilde{N}_{s}(t) .
$$

$\{N(t), t \geq 0\}$ is in general not a Poisson process but rather a compound Poisson process, the time to the $k$ th jump is still $\Gamma(k, 1 / \chi)$-distributed but there are non unit jump sizes. By noting that the probability that the time to the $k$ th loss is less than or equal to $t$ can be expressed as $P(N(t) \geq k)$, it is clear that the distribution of the time to the $k$ th loss can be fully understood from the distribution of $N(t)$ for $t \geq 0$, and this distribution can be evaluated using Panjer recursion or other methods.

\subsection{Panjer Recursion}

If there are common shocks, then $N(t)=\sum_{j=1}^{n} N_{j}(t)$ does not have a Poisson distribution. In our insurance example we have considered only two loss types and it is thus easy to calculate the distribution of $N(t)$ directly using convolution and the bivariate frequency function in (7). A more general method of calculating the probability distribution function of $N(t)$, which will also work in higher dimensional examples, is Panjer recursion (Panjer (1981)). We use the notation of the preceding section. In addition, let $W_{i}$ denote the number of losses due to the $i$ th loss-causing shock. The total number of losses, $N(t)$, has the stochastic representation

$$
N(t) \stackrel{\mathrm{d}}{=} \sum_{k=1}^{\tilde{N}(t)} W_{k},
$$

where $W_{1}, \ldots, W_{\tilde{N}(t)}(\underline{\underline{d}} W)$ are iid and independent of $\tilde{N}(t)$. The probability $P(N(t)=r)$ can now easily be calculated using Panjer recursion. 


\section{Proposition 6.}

$$
P(N(t)=r)= \begin{cases}\sum_{k=1}^{\min (r, n)} \frac{\tilde{\lambda} t k}{r} P(W=k) P(N(t)=r-k), & r \geq 1, \\ \exp \left(-\tilde{\lambda}_{t)},\right. & r=0,\end{cases}
$$

where

$$
P(W=k)= \begin{cases}\chi^{-1} \sum_{e=1}^{m} \lambda^{(e)}\left(\sum_{s:|s|=k} p_{s}^{(e)}+\sum_{i=1}^{n-k}(-1)^{i} \frac{\left(\begin{array}{l}
n \\
k
\end{array}\right)\left(\begin{array}{c}
n-k \\
i
\end{array}\right)}{\left(\begin{array}{c}
n \\
k+i
\end{array}\right)} \sum_{s:|s|=k+i} p_{s}^{(e)}\right), & k<n, \\
\tilde{\lambda}^{-1} \sum_{e=1}^{m} \lambda^{(e)} p_{\{1, \ldots, n\}}^{(e)}, & k=n,\end{cases}
$$

Proof. The formula (11) follows from Theorem 4.4.2, p. 119 in Rolski, Schmidli, Schmidt and Teugels (1998), and that the maximum number of losses due to a loss-causing shock is $n$. The probability that a loss-causing shock causes exactly $k$ losses is given by $P(W=k)=\tilde{\lambda}^{-1} \sum_{s:|s|=k} \lambda_{s}$, where

$$
\sum_{s:|s|=k} \lambda_{s}=\sum_{e=1}^{m} \lambda^{(e)} \sum_{s:|s|=k} \sum_{s^{\prime}: s^{\prime} \supseteq s}(-1)^{\left|s^{\prime}\right|-|s|} p_{s^{\prime}}^{(e)}
$$

The expression of the probability that a loss-causing shock causes $n$ losses can be simplified to

$$
P(W=n)=\tilde{\lambda}^{-1} \sum_{e=1}^{m} \lambda^{(e)} p_{\{1, \ldots, n\}}^{(e)}=\tilde{\lambda}^{-1} \sum_{e=1}^{m} \lambda^{(e)} p_{1, \ldots, n}^{(e)}(1, \ldots, 1) .
$$

For $k \leq n$ we note that there are $\left(\begin{array}{l}n \\ k\end{array}\right)$ sets $s$ with $|s|=k$, and for each such $s$ there are $\left(\begin{array}{c}n-k \\ i\end{array}\right)$ sets of size $k+i(i \in\{1, \ldots, n-k\})$ which contain $s$ as a proper subset. Hence

$$
\sum_{s:|s|=k} \sum_{s^{\prime}: s^{\prime} \supseteq s}(-1)^{\left|s^{\prime}\right|-|s|} p_{s^{\prime}}^{(e)}
$$

consists of $\left(\begin{array}{c}n \\ k\end{array}\right)\left(\begin{array}{c}n-k \\ i\end{array}\right)$ terms $(-1)^{\left|s^{\prime}\right|-|s|} p_{s^{\prime}}^{(e)}$ for which $\left|s^{\prime}\right|=k+i$ and $s \subset s^{\prime}$. Since there are $\left(\begin{array}{c}n \\ k+i\end{array}\right)$ sets $s^{\prime}$ with $\left|s^{\prime}\right|=k+i$ it follows that (12) is equal to

$$
\sum_{s:|s|=k} p_{s}^{(e)}+\sum_{i=1}^{n-k}(-1)^{i} \frac{\left(\begin{array}{c}
n \\
k
\end{array}\right)\left(\begin{array}{c}
n-k \\
i
\end{array}\right)}{\left(\begin{array}{c}
n \\
k+i
\end{array}\right)} \sum_{s:|s|=k+i} p_{s}^{(e)} .
$$


For large $n$, say $n>100$, the usefulness of the Panjer recursion scheme relies heavily on the calculation of $\sum_{s:|s|=k} p_{s}^{(e)}$ for $k \in\{1, \ldots, n\}$. We now look at two specific assumptions on the multivariate Bernoulli distribution of $\mathbf{I}^{(e)}$ conditional on a shock of type $e$. The assumption of conditional independence is attractive for computations since in this case

$$
\sum_{s:|s|=k} p_{s}^{(e)}=\sum_{j_{1}=1}^{n} \sum_{j_{2}>j_{1}} \ldots \sum_{j_{k}>j_{k}-1} p_{j_{1}}^{(e)} p_{j_{2}}^{(e)} \ldots p_{j_{k}}^{(e)} .
$$

Under the assumption of conditional comonotonicity

$$
\sum_{s:|s|=k} p_{s}^{(e)}=\sum_{j_{1}=1}^{n} \sum_{j_{2}>j_{1}} \ldots \sum_{j_{k}>j_{k-1}} \min \left(p_{j_{1}}^{(e)}, p_{j_{2}}^{(e)}, \ldots, p_{j_{k}}^{(e)}\right) .
$$

The latter assumption leads to very efficient computations of $\sum_{s:|s|=k} p_{s}^{(e)}$. Let

$$
\left(p_{\pi_{1}}^{(e)}, p_{\pi_{2}}^{(e)}, \ldots, p_{\pi_{n}}^{(e)}\right)^{\prime}
$$

denote the sorted vector of univariate conditional indicator probabilities, such that $p_{\pi_{1}}^{(e)} \leq p_{\pi_{2}}^{(e)} \leq \ldots \leq p_{\pi_{n}}^{(e)}$. Then

$$
\sum_{s:|s|=k} p_{s}^{(e)}=\sum_{i=1}^{n}\left(\begin{array}{l}
n-i \\
k-1
\end{array}\right) p_{\pi_{i}}^{(e)}
$$

where $\left(\begin{array}{l}n-i \\ k-1\end{array}\right)$ is the number of subsets of size $k$ of $\{1, \ldots, n\}$ with $i$ as smallest element.

\section{The Effect of Dependent Severities}

We now consider adding severities to our shock model and study the multivariate distribution of $\left(Z_{1}(t), \ldots, Z_{n}(t)\right)^{\prime}$. Again we can calculate first and second moments of the marginal distributions and correlations between the components.

\section{Proposition 7.}

1. $\left\{\left(Z_{1}(t), \ldots, Z_{n}(t)\right)^{\prime}, t \geq 0\right\}$ is a multivariate compound Poisson process. If $E\left(\left|X_{j}\right|\right)<\infty$, then

$$
E\left(Z_{j}(t)\right)=E\left(X_{j}\right) E\left(N_{j}(t)\right) .
$$


2. If $E\left(X_{j}^{2}\right), E\left(X_{k}^{2}\right)<\infty$, then the covariance and correlation structure is given by

$$
\operatorname{cov}\left(Z_{j}(t), Z_{k}(t)\right)=E\left(X_{j} X_{k}\right) \operatorname{cov}\left(N_{j}(t), N_{k}(t)\right)
$$

and

$$
\rho\left(Z_{j}(t), Z_{k}(t)\right)=\frac{E\left(X_{j} X_{k}\right)}{\sqrt{E\left(X_{j}^{2}\right) E\left(X_{k}^{2}\right)}} \rho\left(N_{j}(t), N_{k}(t)\right) .
$$

\section{Proof}

1. is easily established from formula (3).

2. We observe that $\forall j, k \in\{1, \ldots, n\}$,

$$
\begin{aligned}
\operatorname{cov}\left(Z_{j}(t), Z_{k}(t)\right)= & \sum_{e=1}^{m} \operatorname{cov}\left(\sum_{r=1}^{N^{(e)}(t)} I_{j, r}^{(e)} X_{j, r}^{(e)}, \sum_{r=1}^{N^{(e)}(t)} I_{k, r}^{(e)} X_{k, r}^{(e)}\right) \\
& +\sum_{e=1}^{m} \sum_{f \neq e} \operatorname{cov}\left(\sum_{r=1}^{N^{(e)}(t)} I_{j, r}^{(e)} X_{j, r}^{(e)}, \sum_{r=1}^{N^{(f)}(t)} I_{k, r}^{(f)} X_{k, r}^{(f)}\right) \\
= & \sum_{e=1}^{m} \operatorname{cov}\left(\sum_{r=1}^{N^{(e)}(t)} I_{j, r}^{(e)} X_{j, r}^{(e)}, \sum_{r=1}^{N^{(e)}(t)} I_{k, r}^{(e)} X_{k, r}^{(e)}\right) \\
= & E\left(X_{j} X_{k}\right) \sum_{e=1}^{m} E\left(N^{(e)}(t)\right) E\left(I_{j}^{(e)} I_{k}^{(e)}\right) \\
= & E\left(X_{j} X_{k}\right) t \sum_{e=1}^{m} \lambda^{(e)} p_{j, k}^{(e)}(1,1) \\
= & E\left(X_{j} X_{k}\right) \operatorname{cov}\left(N_{j}(t), N_{k}(t)\right)
\end{aligned}
$$

Now consider the distribution of the total loss $Z(t)=\sum_{j=1}^{n} Z_{j}(t)$. The expected total loss is easily calculated to be

$$
E(Z(t))=\sum_{j=1}^{n} E\left(X_{j}\right) E\left(N_{j}(t)\right)
$$

and higher moments of $Z(t)$ can also be calculated, by exploiting the compound Poisson nature of this process as shown in (5). Since $\{Z(t), Z \geq 0\}$ is the most general aggregate loss process that we study in this paper we collect some useful moment results for this process. 
Lemma 8. The pth derivative of the characteristic function $\varphi_{Z(t)}$ of $Z(t)$ satisfies

$$
\varphi_{Z(t)}^{(p)}(x)=\lambda t \sum_{k=0}^{p-1}\left(\begin{array}{c}
p-1 \\
k
\end{array}\right) \varphi_{Y}^{(k+1)}(x) \varphi_{Z(t)}^{(p-k-1)}(x),
$$

where $\varphi_{Y}$ denotes the characteristic function of $Y$.

Proof. The formula (13) is proved by induction using the binomial relation $\left(\begin{array}{c}p-1 \\ k\end{array}\right)+\left(\begin{array}{c}p-1 \\ k+1\end{array}\right)=\left(\begin{array}{c}p \\ k+1\end{array}\right)$. The induction step reads as follows. Fix $p$ and suppose that (13) holds. Then

$$
\begin{aligned}
\varphi_{Z(t)}^{(p+1)}(x)= & \lambda t \sum_{k=0}^{p-1}\left(\begin{array}{c}
p-1 \\
k
\end{array}\right)\left(\varphi_{Y}^{(k+2)}(x) \varphi_{Z(t)}^{(p-k-1)}(x)+\varphi_{Y}^{(k+1)}(x) \varphi_{Z(t)}^{(p-k)}(x)\right) \\
= & \lambda t \sum_{k=0}^{p-2}\left(\begin{array}{c}
p-1 \\
k
\end{array}\right) \varphi_{Y}^{(k+2)}(x) \varphi_{Z(t)}^{(p-k-1)}(x)+\lambda t \varphi_{Y}^{(p+1)}(x) \varphi_{Z(t)}^{(0)}(x) \\
& +\lambda t \varphi_{Y}^{(1)}(x) \varphi_{Z(t)}^{(p)}(x)+\lambda t \sum_{k=0}^{p-2}\left(\begin{array}{c}
p-1 \\
k+1
\end{array}\right) \varphi_{Y}^{(k+2)}(x) \varphi_{Z(t)}^{(p-k-1)}(x) \\
= & \lambda t \varphi_{Y}^{(1)}(x) \varphi_{Z(t)}^{(p)}(x)+\lambda t \sum_{k=1}^{p-1}\left(\begin{array}{l}
p \\
k
\end{array}\right) \varphi_{Y}^{(k+1)}(x) \varphi_{Z(t)}^{(p-k)}(x) \\
& +\lambda t \varphi_{Y}^{(p+1)}(x) \varphi_{Z(t)}^{(0)}(x) \\
= & \lambda t \sum_{k=0}^{p}\left(\begin{array}{l}
p \\
k
\end{array}\right) \varphi_{Y}^{(k+1)}(x) \varphi_{Z(t)}^{(p-k)}(x)
\end{aligned}
$$

Using Lemma 8, the moments of $Z(t)$ can be calculated as follows.

\section{Proposition 9.}

1. If they exist, the 2 nd and $3 r d$ order central moment of $Z(t)$ are given by

$$
E\left((Z(t)-E(Z(t)))^{p}\right)=\lambda t E\left(Y^{p}\right), \quad p=2,3,
$$

where $\lambda=\sum_{e=1}^{m} \lambda^{(e)}$ and

$$
E\left(Y^{p}\right)=\frac{1}{\lambda} \sum_{j_{1}=1}^{n} \ldots \sum_{j_{p}=1}^{n} E\left(X_{j_{1}} \ldots X_{j_{p}}\right) \sum_{e=1}^{m} \lambda^{(e)} p_{j_{1}, \ldots, j_{p}}^{(e)}(1, \ldots, 1) .
$$

2. Whenever they exist, the non-central moments of $Z(t)$ are given recursively by

$$
E\left(Z(t)^{p}\right)=\lambda t \sum_{k=0}^{p-1}\left(\begin{array}{c}
p-1 \\
k
\end{array}\right) E\left(Y^{k+1}\right) E\left(Z(t)^{p-k-1}\right),
$$

with $E\left(Y^{k+1}\right)$ given by (15). 


\section{Proof}

1. For a compound Poisson process of the form (5) the formula (14) is well known. We can calculate that for all $p$

$$
\begin{aligned}
E\left(Y^{p}\right) & =\sum_{e=1}^{m} \frac{\lambda^{(e)}}{\lambda} E\left(\left(\mathbf{I}^{(e)} \mathbf{X}\right)^{p}\right) \\
& =\sum_{e=1}^{m} \frac{\lambda^{(e)}}{\lambda} E\left(\sum_{j_{1}=1}^{n} \ldots \sum_{j_{p}=1}^{n} I_{j_{1}}^{(e)} \ldots I_{j_{p}}^{(e)} X_{j_{1}} \ldots X_{j_{p}}\right) \\
& =\lambda^{-1} \sum_{e=1}^{m} \lambda^{(e)} \sum_{j_{1}=1}^{n} \ldots \sum_{j_{p}=1}^{n} E\left(X_{j_{1}} \ldots X_{j_{p}}\right) p_{j_{1}, \ldots, j_{p}}^{(e)}(1, \ldots, 1) \\
& =\lambda^{-1} \sum_{j_{1}=1}^{n} \ldots \sum_{j_{p}=1}^{n} E\left(X_{j_{1}} \ldots X_{j_{p}}\right) \sum_{e=1}^{m} \lambda^{(e)} p_{j_{1}, \ldots, j_{p}}^{(e)}(1, \ldots, 1) .
\end{aligned}
$$

2. If $E\left(|Z(t)|^{p}\right)<\infty$, then $\varphi_{Z(t)}^{(k)}(0)=i^{k} E\left(Z(t)^{k}\right)$ for $k=1, \ldots, p$. The conclusion follows by applying this to (13).

We are particularly interested in the effect of different levels of dependence between both loss frequencies and loss severities on the tail of the distribution of $Z(t)$, and on higher quantiles of this distribution. The distribution of $Z(t)$ is generally not available analytically but, given the ease of simulating from our Poisson common shock model, it is possible to estimate quantiles empirically to a high enough degree of accuracy that differences between different dependence specifications become apparent.

It is also possible, given the ease of calculating moments of $Z(t)$, to use a moment fitting approach to approximate the distribution of $Z(t)$ with various parametric distributions, and we implement this approach in the following example.

\subsection{Insurance example (continued)}

Assume that French and German severities are Pareto(4,3) distributed, i.e. $F_{i}(x)=P\left(X_{i} \leq x\right)=1-\left(\frac{3}{3+x}\right)^{4}, E\left(X_{i}\right)=1, E\left(X_{i}^{2}\right)=3, E\left(X_{i}^{3}\right)=27, i=1,2$.

We have to fix the dependence structure of potential losses $\left(X_{1}, X_{2}\right)^{\prime}$ at the same shock. We do this using the copula approach. The copula $C$ of $\left(X_{1}, X_{2}\right)^{\prime}$ is the distribution function of $\left(F_{1}\left(X_{1}\right), F_{2}\left(X_{2}\right)\right)^{\prime}$. The distribution function of $\left(X_{1}, X_{2}\right)^{\prime}$ can be expressed in terms of $C$ as

$$
F\left(x_{1}, x_{2}\right)=C\left(F_{1}\left(x_{1}\right), F_{2}\left(x_{2}\right)\right) \text {. }
$$


For more on copulas see Embrechts, McNeil and Straumann (2001), Nelsen (1999) or Joe (1997). We consider three cases.

- Independent severities:

$$
F\left(x_{1}, x_{2}\right)=F_{1}\left(x_{1}\right) F_{2}\left(x_{2}\right) .
$$

- Positively dependent severities with Gaussian dependence:

$$
F\left(x_{1}, x_{2}\right)=C_{\rho}^{\mathrm{Ga}}\left(F_{1}\left(x_{1}\right), F_{2}\left(x_{2}\right)\right),
$$

where

$$
\begin{aligned}
& C_{\rho}^{\mathrm{Ga}}(u, v)=\int_{-\infty}^{\Phi^{-1}(u)} \int_{-\infty}^{\Phi^{-1}(v)} \frac{1}{2 \pi\left(1-\rho^{2}\right)^{1 / 2}} \exp \left\{\frac{-\left(s^{2}-2 \rho s t+t^{2}\right)}{2\left(1-\rho^{2}\right)}\right\} d s d t . \\
& \text { and } \rho \in(0,1) \text {. }
\end{aligned}
$$

- Positively dependent severities with Gumbel dependence:

$$
F\left(x_{1}, x_{2}\right)=C_{\theta}^{\mathrm{Gu}}\left(F_{1}\left(x_{1}\right), F_{2}\left(x_{2}\right)\right),
$$

where

$$
C_{\theta}^{\mathrm{Gu}}(u, v)=\exp \left(-\left\{(-\log u)^{\theta}+(-\log v)^{\theta}\right\}^{1 / \theta}\right),
$$

and $\theta>1$.

For both of the positive dependence models we will parameterize the copulas such that Kendall's rank correlation $(\tau)$ (see e.g. Embrechts, McNeil and Straumann (2001) for details) between $X_{1}$ and $X_{2}$ is 0.5 . This is achieved by setting

$$
\rho=\sin \left(\frac{\pi}{2} \tau\right) \text { and } \theta=\frac{1}{1-\tau} .
$$

As we have discussed there are several possibilities for modelling the tail of $Z(5)$. One approach is to fit a heavy-tailed generalised F-distribution (referred to as a generalised Pareto distribution in Hogg and Klugman (1994)) to $Z(5)$ using moment fitting with the first three moments. The distribution function is given by

$$
H_{\alpha, \lambda, k}(x)=G\left(2 k, 2 \alpha, \frac{\alpha}{k \lambda} x\right) \text { for } \alpha>0, \lambda>0, \mathrm{k}>0,
$$

where $G\left(v_{1}, v_{2}, \cdot\right)$ is the distribution function for the F-distribution with $v_{1}$ and $v_{2}$ degrees of freedom. The $n$th moment exists if $\alpha>n$ and is then given by

$$
\lambda^{n}\left(\prod_{i-0}^{n-1}(k+i)\right) /\left(\prod_{i=1}^{n}(\alpha-i)\right) .
$$


By calculating the first three moments of $Z(5)$ for different frequency and severity dependencies we fit generalised F-distributions and study the difference in tail behaviour. Figure 2 shows quantiles of generalised F-distributions determined by fitting the first three moments to $Z(5)$ for case 1,2 and 3 and for different dependence structures between the severities. It clearly shows the effect of common shocks on the tail of $Z(5)$ and perhaps even more the drastic change in tail behaviour when adding moderate dependence between the severities.
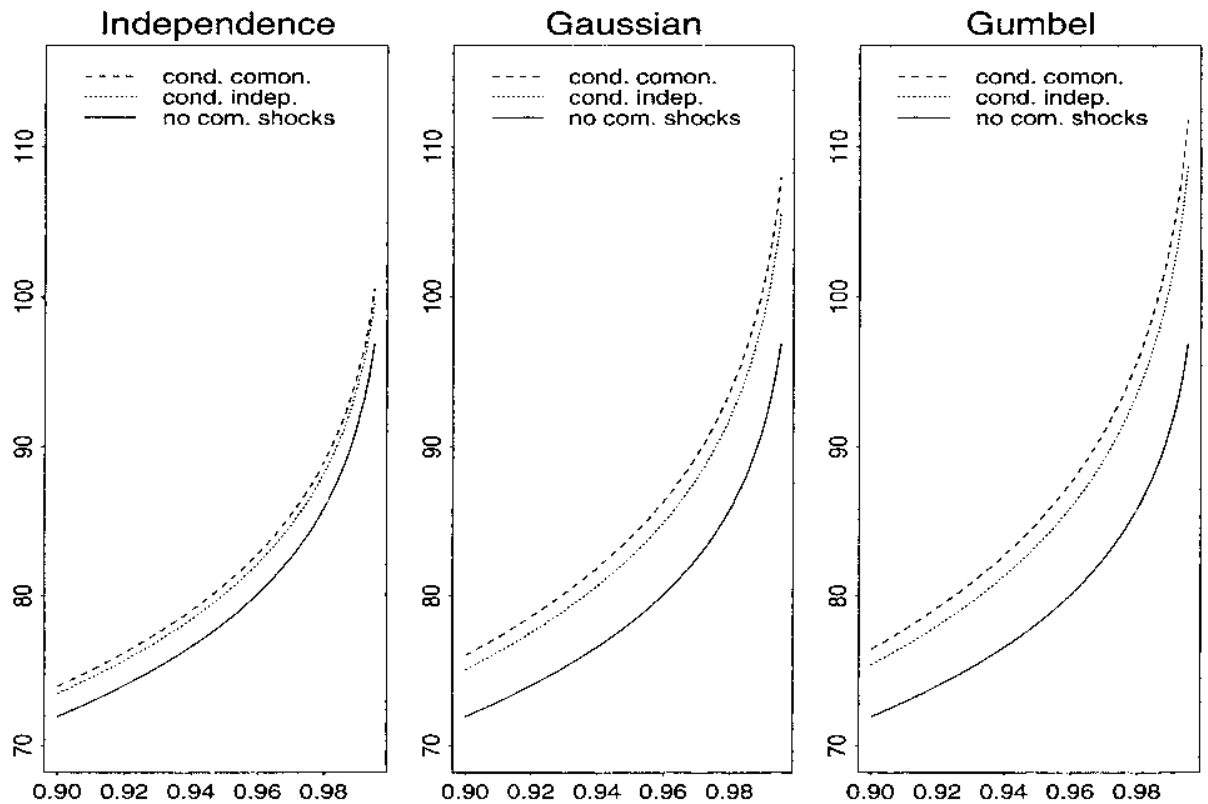

Figure 2: The curves from lower to upper show the quantiles $H_{\alpha, \lambda, k}^{-1}(q)$ of moment fitted generalised F-distributions for case 1,2 and 3 and $q \in[0.900,0.995]$. The first three moments coincide with those of $Z(5)$.

It should be noted that the quantile estimates of $Z(5)$ given by moment fitted generalised F-distributions are slight overestimates of the true quantiles for $\alpha \in[0.900,0.995]$. However the accuracy is sufficient to show the major differences between the quantile curves of $Z(5)$ for our different copula choices.

\section{Applying the Methodology to Portfolio Credit Risk}

We consider here the problem of quantifying risk in large portfolios of defaultable assets, the simplest example being loan portfolios. Models that are used for this purpose address the phenomenon of dependent defaults and a number 
of approaches have been suggested in the literature in recent years and implemented in widely-used industry solutions such as the model proposed by the KMV corporation (KMV-Corporation 1997), the model of the RiskMetrics group (RiskMetrics-Group 1997), or CreditRisk ${ }^{+}$, developed by Credit Suisse Financial Products (Credit-Suisse-Financial-Products 1997). In this section we describe how the common Poisson shock model provides a simple alternative framework for modelling portfolio credit risk.

We emphasise that our focus here, and that of the industry models, is on overall portfolio credit risk. We are less concerned with providing detailed analysis of the individual default potential of a single credit risk, such as is required in the pricing of defaultable bonds or standard credit derivatives. For this purpose the class of default intensity models has emerged as the most important; see for example Jarrow and Turnbull (1995).

\subsection{Poisson shock models for defaults}

We consider a portfolio of loans and develop a shock model by considering that every counterparty in the portfolio defines a loss type and that a variety of different kinds of economic shock may lead to the default of these counterparties: global shock events may potentially affect all counterparties; sector shock events may affect only certain kinds of company, such as companies in a particular geographical area or companies concentrated on a particular industry; idiosyncratic shock events (such as an episode of bad management) may affect only individual counterparties; one might also think of endogenous shock events where the default of important primary counterparties might affect other counterparties, so that default was contagious.

In all cases the common shock construction means that defaults of individual counterparties are modelled by the first events in a series of dependent Poisson process. Suppose the random vector $\left.\mathbf{T}=T_{1}, \ldots, T_{n}\right)^{\prime}$ describes the times to default for the $n$ counterparties in the portfolio. In Section 3.2 we observed that this vector of default times has a multivariate exponential distribution with the Marshall-Olkin survival copula. Suppose that time is measured in years and that we are interested in the portfolio credit loss distribution for a time horizon of 1 year; suppose further that the exposures (loan sizes) are known and given by $e_{1}, \ldots, e_{n}$. If we neglect interest rates and assume that nothing is recovered from defaulted firms then the overall portfolio loss is given by

$$
L=\sum_{i=1}^{n} e_{i} 1_{\left\{T_{i} \leq 1\right\}}
$$

Frey and McNeil (2001) have shown that the distribution of L is fully determined by the set of individual default probabilities $\left\{p_{i}=P\left(T_{i} \leq 1\right), i=1, \ldots, n\right\}$ and the copula $C$ of the vector $\mathbf{T}$; the exponential distributional form for the margins of $\mathbf{T}$ is not a critical feature of the model. They also show that the tail of the distribution of $L$ and related risk measures are often much more sensitive 
to the assumptions about the dependence between the defaults as summarised by the copula, than they are to the accurate specification of individual default probabilities.

\subsection{Relation of the shock model to standard models}

All other models that have been suggested for portfolio credit risk also imply multivariate distributions for the vector of default times of the portfolio members. Regardless of whether these models are set up as one-period or multi-period models, or whether they assume constant intensities of default (as in the Poisson shock model), non-homogeneous intensities or stochastic intensities, they all imply a distribution for the counterparty survival times. From model to model, these distributions will vary with respect to both their marginal distributions and their copulas, but as far as determining the loss distribution is concerned, if they have been calibrated to give broadly similar individual default probabilities it is the copula that will be decisive in determining the tail of the portfolio loss distribution.

Both KMV and CreditMetrics may be considered to descend from the firmvalue model of Merton (1974), where default is modelled as occurring when the asset value of a company falls below its liabilities, and asset value changes are considered to have a multivariate normal distribution. Although this appears very different to the Poisson shock model, as far as the loss distribution for a fixed time horizon is concerned, both KMV and CreditMetrics are in fact structurally equivalent to a model in which default times have a multivariate exponential distribution with the Gaussian copula (i.e. the copula that describes the dependence inherent in a multivariate normal distribution); see Li (1999) and Frey and McNeil (2001) for more detail. Thus the crucial difference in the one-period framework lies in the fact that these industry models imply a Gaussian copula whereas common shocks imply a Marshall-Olkin copula to describe the dependence of the survival times.

The CreditRisk+ model employs a mixture-modelling philosophy which assumes that conditional on a vector of independent gamma-distributed macroeconomic factors, the default of a counterparty occurs independently of other counterparties and is the first event in a Poisson process with an intensity that depends on these factors. Survival times are not exponential (but rather conditionally exponential), but for portfolio risk modelling in a one-period setting, this is again not a decisive factor. Assuming that the model has been calibrated to give plausible values for individual default probabilities, it is the copula of the default times that is most important in determining the overall loss distribution, although this copula is difficult to isolate in closed form in the general version of CreditRisk+.

There have been a number of papers on the subject of extending the intensity-based approach to modelling the default of single counterparties to obtain models for dependent defaults of several counterparties, principally with the problem of pricing so-called basket credit derivatives in mind; see Schoenbucher and Schubert (2001) for a useful summary of these approaches. 
We mention in particular a model of Duffie and Singleton (1995) where individual defaults follow Cox processes with stochastic intensities. These intensities may jump (by a random amount) when certain common shocks occur which have the potential to affect all counterparties, or when idiosyncratic shocks occur affecting an individual company; as in our model shocks occur as Poisson processes. Our model can be thought of a cruder version of the Duffie \& Singleton model with constant and deterministic intensities for individual defaults.

\subsection{Setting up the shock model}

Consider a loan portfolio consisting of $n$ obligors. Suppose the counterparties can be divided into $K$ geographical or industry sectors. We consider a model where obligors are subject to idiosyncratic, sector and global shocks, so that there are a total of $m=n+K+1$ shock event processes.

Suppose that the $j$ th obligor belongs to sector $k=k_{j}$ where $k \in\{1, \ldots, K\}$. From formula (6) we know that $N_{j}(t)$, the number of defaults of obligor $j$ in $(0, t]$ is Poisson with intensity given by

$$
\lambda_{j}=\lambda^{(j)}+p_{j}^{(n+k)} \lambda^{(n+k)}+p_{j}^{(m)} \lambda^{(m)}, \quad k=k(j),
$$

where the three terms represent the contributions to the default intensity of idiosyncratic, sector and global events respectively. Note that in general this intensity will be set so low that the probability of a firm defaulting more than once in the period of interest can be considered negligible.

This is a very general model and to obtain a model that we would have a hope of calibrating in a real application we need to drastically reduce the number of parameters in the model. We assume first that companies can be grouped together into rating classes within which default rates can be considered constant and known. It is very common in portfolio default risk modelling to base the assessment of default intensities for individual companies on information about historical default rates for similarly rated companies. Suppose that the $j$ th obligor belongs to rating category $l=l(j)$ where $l \in\{1, \ldots, L\}$. We assume for the overall default intensity $\lambda_{j}$ that

$$
\lambda_{j}=\lambda_{\text {total }, l}, \quad l=l(j), j=1, \ldots, n .
$$

To achieve (16) we assume that the rate of occurrence of idiosyncratic shocks also depends only on the rating category and we adopt the notation

$$
\begin{aligned}
& \lambda^{(j)}=\lambda_{\text {idio, } l}, \quad l=l(j), j=1, \ldots, n, \\
& \lambda^{(n+k)}=\lambda_{\text {sector }, k}, \quad k=1, \ldots, K, \\
& \lambda^{(m)}=\lambda_{\text {global }} .
\end{aligned}
$$


Clearly we now have a total of $L+K+1$ shock intensities to set.

We assume also that the conditional default probabilities given the occurrence of sector shocks only depend on the rating class of the company and write for an obligor $j$

$$
p_{j}^{(n+k)}=s_{k, l}, \quad l=l(j) .
$$

We assume moreover that the default indicators for several companies in the same sector are conditionally independent given the occurrence of an event in that sector. Analogously, we assume that the conditional default probabilities given the occurrence of global shocks depend on both rating class and sector of the company and write

$$
p_{j}^{(m)}=g_{k, l}, \quad l=l(j), k=k(j) .
$$

We assume that the default indicators for any group of companies are conditionally independent given the occurrence of a global event.

In total we have $2 K L$ conditional default probabilities to set and we have the system of equations

$$
\lambda_{\text {total }, l}=\lambda_{\text {idio }, l}+s_{k, l} \lambda_{\text {sector }, k}+g_{k, l} \lambda_{\text {global }}, k=1, \ldots, K, l=1, \ldots, L,
$$

subject to the constraint, imposed by (16), that

$$
s_{k, l} \lambda_{\text {sector }, k}+g_{k, l} \lambda_{\text {global }}=s_{k^{\prime}, l} \lambda_{\text {sector }, k^{\prime}}+g_{k^{\prime}, l} \lambda_{\text {global }}, \forall k \neq k^{\prime} .
$$

\subsection{Understanding the factors determining the risk}

We are interested in the behaviour of $N(t)$, the total number of defaults, for fixed $t$. If we suppose that the individual default rates have been fixed then $E(N(t))$ has been fixed. However, depending how we set the various shock intensities and individual default probabilities the risk inherent in $N(t)$ may vary considerably. If we measure risk by variance we can get analytically an idea of which factors affect the risk by considering

$$
\operatorname{var}(N(t))-E(N(t))=\sum_{j_{1}, j_{2}: j_{1} \neq j_{2}} \operatorname{cov}\left(N_{j_{1}}(t), N_{j_{2}}(t)\right) .
$$

For simplicity we set $t=1$ and consider a model with one rating class $(L=1)$ and assume that the conditional default probabilities do not depend on the sector for all global shocks $\left(g_{k, 1}=g, \forall k\right)$. Let there be $n_{k}$ obligors in sector $k$. We have for $j_{1} \neq j_{2}$ that

$$
\operatorname{cov}\left(N_{j_{1}}(1), N_{j_{2}}(1)\right)= \begin{cases}g^{2} \lambda_{\text {global }} & k\left(j_{1}\right) \neq k\left(j_{2}\right), \\ g^{2} \lambda_{\text {global }}+s_{k}^{2} \lambda_{\text {sector }, k} & k\left(j_{1}\right)=k\left(j_{2}\right)=k,\end{cases}
$$


which allows us to calculate that

$$
\operatorname{var}(N(1))-E(N(1))=\left(n^{2}-n\right) g^{2} \lambda_{\text {global }}+\sum_{k=1}^{K}\left(n_{k}^{2}-n_{k}\right) s_{k}^{2} \lambda_{\text {sector } k} .
$$

In view of (17) we have that $s_{k} \lambda_{\text {sector } k}$, the intensity of default due to sector shocks in sector $k$, must be equal for all $k$ in this special case. If we write $\delta_{\text {global }}=g \lambda_{\text {global }}$ and $\delta_{\text {sector }}=s_{k} \lambda_{\text {sector }, k}, \forall k$, for the default intensities due to global or sector causes we obtain finally

$$
\operatorname{var}(N(1))-E(N(1))=\delta_{\text {global }}\left(n^{2}-n\right) g+\delta_{\text {sector }} \sum_{k=1}^{K}\left(n_{k}^{2}-n_{k}\right) s_{k} .
$$

This expression allows us to draw two broad conclusions about the riskiness of the model as measured by the variance of the number of defaults.

- The higher the portion of the default intensity that we attribute to sector and global shocks (common shocks) the riskier the model. As before, the only way we can have a Poisson distribution for $N(1)$ is if the default intensity can be attributed entirely to idiosyncratic shocks.

- Suppose we assume common shocks and fix the portions of the default intensity that we attribute to common shocks $\left(\delta_{\text {global }}\right.$ and $\left.\delta_{\text {sector }}\right)$. The overall risk also depends on how we set the conditional default probabilities $g$ and $s_{k}$. Low shock intensities and high conditional default probabilities are riskier than the other way around.

These conclusions are confirmed in the following simulation example where we allow two rating categories and more heterogeneous conditional default probabilities.

\subsection{A simulation study}

In our examples we take $t=1$ year and consider $K=4$ sectors and $L=2$ rating categories; we assume that overall default rates for these categories are $\lambda_{\text {total }, 1}=0.005$ and $\lambda_{\text {total }, 2}=0.02$. Let $n_{k, l}$ denote the number of companies in rating class $l$ and sector $k$. We set

$$
\begin{aligned}
& n_{1,1}=10000, n_{2,1}=20000, n_{3,1}=15000, n_{4,1}=5000, \\
& n_{1,2}=10000, n_{2,2}=25000, n_{3,2}=10000, n_{4,2}=5000 .
\end{aligned}
$$

In the following two cases we investigate the sensitivity of the tail of $N(1)$ to the specification of model parameters. Results are based on 10000 simulated realizations of $N(1)$ and models are compared with respect to estimates of the $95 \%$ and $99 \%$ quantiles.

\section{- Case 1}

We study the effects of increasing the intensity of the common shocks and decreasing the intensity of the idiosyncratic shocks when the univariate 
conditional default probabilities are held constant. We set the values of these parameters to be

$$
\begin{gathered}
\left(s_{1,1}, s_{2,1}, s_{3,1}, s_{4,1}, s_{1,2}, s_{2,2}, s_{3,1}, s_{4,2}\right) \\
=(0.25,0.08,0.05,0.1,1,0.3,0.25,0.25) 10^{-2} \\
\left(g_{1,1}, g_{2,1}, g_{3,1}, g_{4,1}, g_{1,2}, g_{2,2}, g_{3,1}, g_{4,2}\right) \\
=(0.25,0.1,0.4,0.1,1,0.5,1.5,1) 10^{-2} .
\end{gathered}
$$

We have some flexibility in choosing the intensities

$$
\begin{aligned}
& (0.005,0.02,0.0,0.0,0.0,0.0,0.0) \rightarrow(0.004,0.016,0.2,1.0,0.4,0.8,0.2) \rightarrow \\
& (0.002,0.008,0.6,3.0,1.2,2.4,0.6) \rightarrow(0.0,0.0,1.0,5.0,2.0,4.0,1.0)) .
\end{aligned}
$$

Hence we start with the special case of no common shocks and a situation where every individual default process $N_{j}(t)$ is independent Poisson and the total number of defaults $N(t)$ is Poisson. In the second model we still attribute $80 \%$ of the default intensities $\lambda_{j}$ to idiosyncratic shocks, but we now have $20 \%$ in common shocks. In the third model we have $60 \%$ in common shocks and in the final model we have only common shocks. The effect of the increasing portion of defaults due to common shocks on the distribution of $N(1)$ is seen in Figure 4 and empirical quantiles of $N(1)$ are given in Table 1.

\section{- Case 2}

Suppose we attribute $40 \%$ of defaults for companies in both ratings classes to idiosyncratic shocks and $60 \%$ to common shocks. That is we assume

$$
\left(\lambda_{\text {idio, }, 1}, \lambda_{\text {idio, } 2}\right)=(0.002,0.008) \text {. }
$$

Suppose, for both rating classes, we attribute to sector specific causes, $20 \%$ of defaults of sector 1 companies, $50 \%$ of defaults of sector 2 companies, $10 \%$ of defaults of sector 3 companies and $40 \%$ of defaults of sector 4 companies. Moreover we believe that the frequencies of sector and global shocks are in the ratio

$$
\lambda_{\text {sector }, 1}: \lambda_{\text {sector }, 2}: \lambda_{\text {sector }, 3}: \lambda_{\text {sector, } 4}: \lambda_{\text {global }}=1: 5: 2: 4: 1
$$

We have now specified the model up to a single factor $f$. For any $f \geq 0.05$ the following choices of model parameters would satisfy our requirements

$$
\begin{aligned}
& \left(\lambda_{\text {sector }, 1}, \lambda_{\text {sector }, 2}, \lambda_{\text {sector }, 3}, \lambda_{\text {sector }, 4}, \lambda_{\text {global }}\right) \\
& \quad=f(0.2,1.0,0.4,0.8,0.2) \\
& \left(s_{1,1}, s_{2,1}, s_{3,1}, s_{4,1}, s_{1,2}, s_{2,2}, s_{3,1}, s_{4,2}\right) \\
& \quad=\frac{1}{f}(0.5,0.25,0.125,0.25,2,1,0.5,1) 10^{-2}
\end{aligned}
$$




$$
\begin{aligned}
& \left(g_{1,1}, g_{2,1}, g_{3,1}, g_{4,1}, g_{1,2}, g_{2,2}, g_{3,1}, g_{4,2}\right) \\
& \quad=\frac{1}{f}(1,0.25,1.25,0.5,4,1,5,2) 10^{-2} .
\end{aligned}
$$

The condition $f \geq 0.05$ is to ensure that $s_{1}^{1}, \ldots, s_{2}^{4}, g_{1}^{1}, \ldots, g_{2}^{4} \leq 1$. When $f$ is increased by a factor $\Delta f$ the intensities of the common shocks are increased by a factor $\Delta f$ and the univariate conditional default probabilities are decreased by a factor $1 / \Delta f$. The effect of increasing $f$ on the distribution of $N(1)$ is seen in figure 3 , where histograms are plotted by row for $f=1,2,4,8$. The key message is as anticipated that low shock intensities and high conditional default probabilities are more riskier than the other way around. Values for the empirical 95th and 99th percentiles of the distribution of $N(1)$ are given in Table 1.
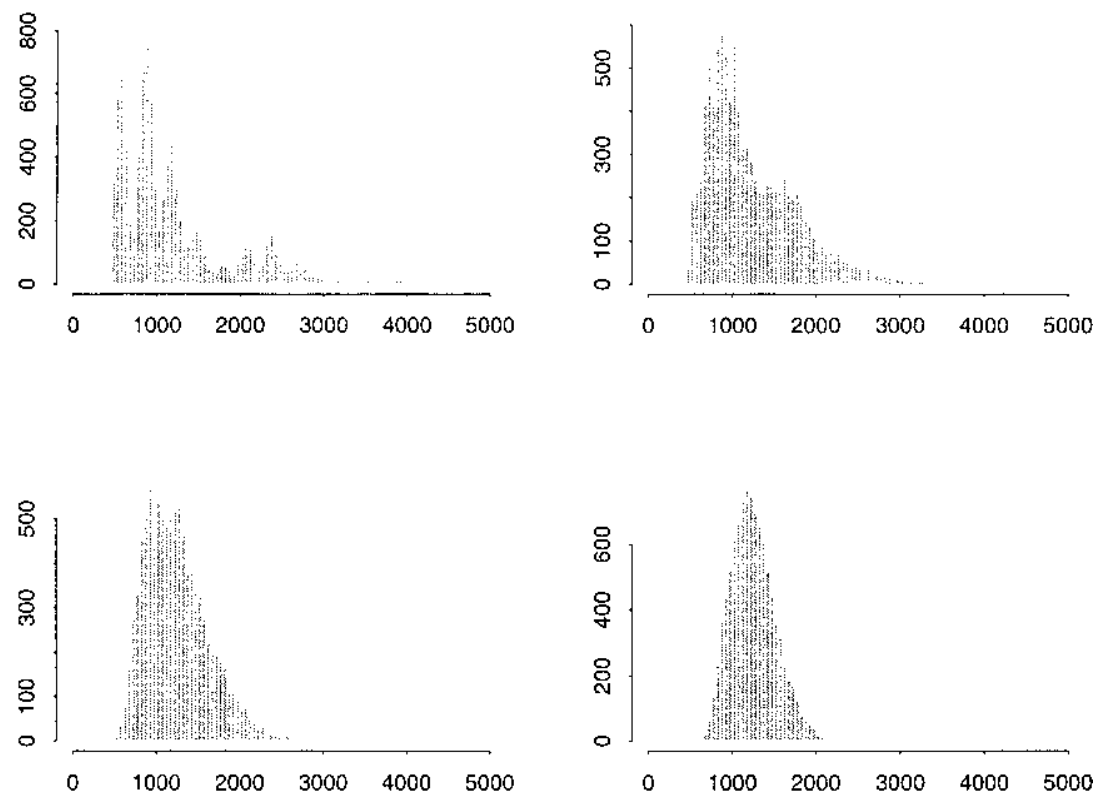

Figure 3: Histograms of 10000 independent simulations of $N(1)$, the number of defaults in a one year period, for $f=1$ (upper left), $f=2$ (upper right), $f=4$ (lower left) and $f=8$ (lower right).

TABLE 1

EMPIRICAL QUANTILES OF $N(1)$ CORRESPONDING TO THE SAMPLES OF SIZE 10000 SHOWN IN FIGURES 3 AND 4.

\begin{tabular}{lllllllll}
\hline \hline & \multicolumn{4}{c}{ Case $\mathbf{1}$} & \multicolumn{4}{c}{ Case 2 } \\
& $\boldsymbol{f = 1}$ & $\boldsymbol{f = 2}$ & $\boldsymbol{f}=\mathbf{4}$ & $\boldsymbol{f =}$ & $\boldsymbol{f}=\mathbf{1}$ & $\boldsymbol{f}=\mathbf{2}$ & $\boldsymbol{f = 4}$ & $\boldsymbol{f}=\boldsymbol{8}$ \\
\hline$a=0.95$ & 2742 & 2307 & 1957 & 1734 & 1308 & 1769 & 2106 & 2346 \\
$a=0.99$ & 3898 & 2889 & 2381 & 1972 & 1331 & 2180 & 2622 & 2948 \\
\hline \hline
\end{tabular}



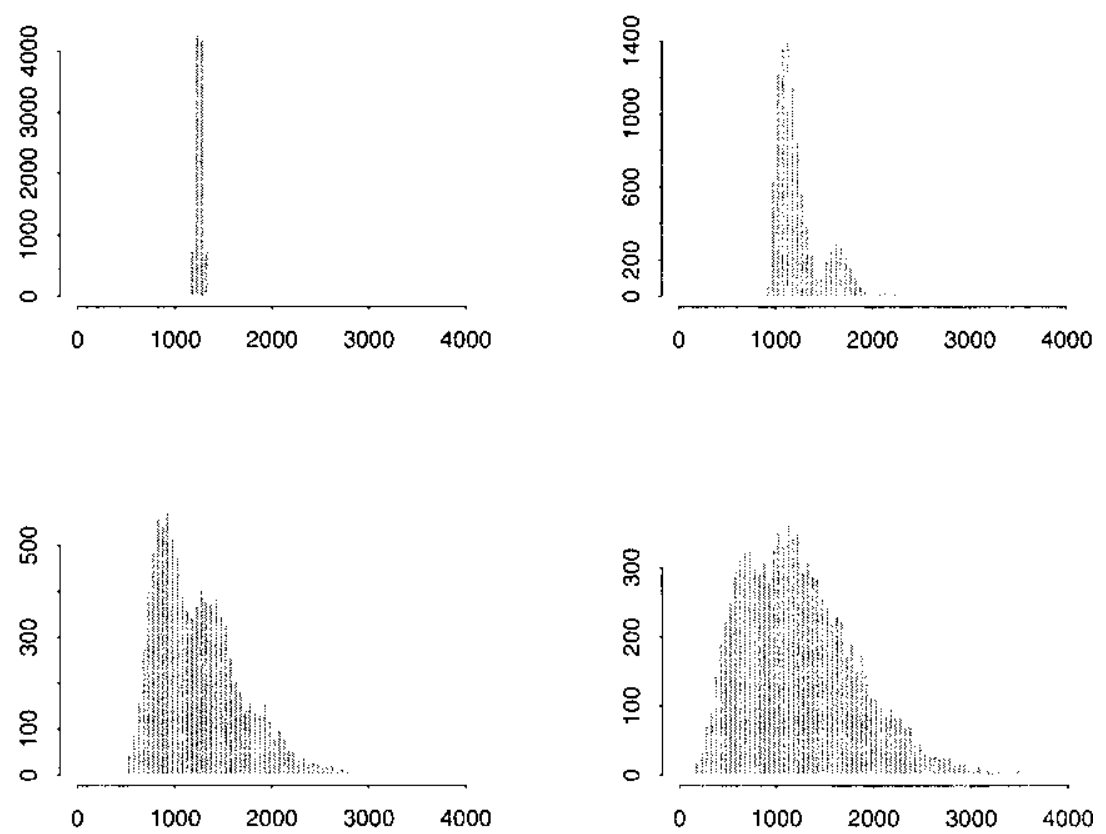

Figure 4: Histograms of 10000 independent simulations of $N(1)$ when increasing the intensities of the common shocks and decreasing the intensities of the idiosyncratic shocks while holding the univariate conditional default probabilities fixed. (1) upper left, (2) upper right, (3) lower left, (4) lower right.

\subsection{Conclusion}

Clearly the calibration of such models is a difficult and highly judgemental enterprise and the method would seem most useful as a broad brush approach to assessing the risk of a portfolio about which relatively little is known; it might be useful for instance in generating possible future loss scenarios under a variety of assumptions about the frequency and severity of economic downturns. Obviously the higher the number of rating classes and sectors that are introduced the more difficult the calibration will prove to be. Our analysis in Section 5.4 and our simulations in Section 5.5 suggest that calibration might proceed along the following lines.

1. For each combination of rating class and sector, historical data on defaults should be used to estimate what proportions can be attributed to idiosyncratic, sector or global causes. In determining these proportions the constraints imposed by (16) must be respected.

2. Having carved up the individual intensities into these three portions we should then attempt to determine the relative intensity of sector and global events.

3. Bearing in mind that the conditional default probabilities can have a profound impact on the loss distribution we now fix the absolute intensity of sector and global shocks as well as these conditional default probabilities; 
for conservatism we err on the side of underestimating shock intensities and overestimating conditional default probabilities.

\section{REFERENCES}

Barlow, R., and Proschan, F. (1975) Statistical Theory of Reliability and Life Testing. Holt, Rinehart \& Winston, New York.

Credit-Suisse-Financial-Products (1997) CreditRisk ${ }^{+}$a Credit Risk Management Framework. Technical Document, available from htpp://www.csfb.com/creditrisk.

Duffie, D., and Singleton, K. (1998) Simulating Correlated Defaults. Working paper, Graduate School of Business, Stanford University.

Embrechts, P., McNeil, A., and StraumanN, D. (2001) Correlation and dependency in risk management: properties and pitfalls. In Risk Management: Value at Risk and Beyond, ed. by M. Dempster. Cambridge University Press, Cambridge.

Frey, R., and McNeIL, A. (2001): Modelling dependent defaults. Preprint, ETH Zürich, available from http://www.math.ethz.ch/ frey.

HogG, R., and Klugman, S. (1984) Loss Distributions. Wiley, New York.

Jarrow, R., and Turnbull, S. (1995) Pricing Derivatives on Financial Securities Subject to Credit Risk. Journal of Finance, L(1), 83-85.

Joe, R. (1997) Multivariate Models and Dependence Concepts. Chapman \& Hall, London.

KMV-Corporation (1997) Modelling Default Risk. Technical Document, available from http:// www.kmv.com.

LI, D. (1999) On Default Correlation: A Copula Function Approach. Working paper, RiskMetrics Group, New York.

LI, H., and XU, S. (2001) Stochastic bounds and dependence properties of survival times in a multicomponent shock model. Journal of Multivariate Analysis 76, 63-89.

Marshall, A., and Olkin, I. (1967) A multivariate exponential distribution. Journal of American Statistical Association 62, 30-44.

Merton, R. (1974) On the Pricing of Corporate Debt: The Risk Structure of Interest Rates. Journal of Finance 29, 449-470.

Nelsen, R.B. (1999) An Introduction to Copulas. Springer, New York.

PANJER, H. (1981) Recursive evaluation of a family of compound distributions. ASTIN Bulletin 12, 22-26.

RiskMetrics-Group (1997) CreditMetrics - Technical Document, available from http://www. riskmetrics.com/research.

Rolski, T., Schmidli, H., Schmidt, V., and Teugels, J. (1998) Stochastic Processes for Insurance and Finance. Wiley, Chichester.

SAvits, T. (1988) Some multivariate distributions derived from a non-fatal shock model. Journal of Applied Probability 25, 383-390.

SCHÖnbuCHER, P., and SCHUbeRT, D. (2001) Copula-dependent default risk in intensity models. Working paper.

Wang, S., and Dhaene, J. (1998) Comonotonicity, correlation order and premium principles. Insurance: Mathematics and Economics 22, 235-242.

FILIP LINDSKOG

Risklab

Federal Institute of Technology

ETH Zentrum

CH-8092 Zurich

Tel.: +4116326741

Tel.: +4116321085

lindskog@math.ethz.ch 


\author{
Alexander J. McNeIL \\ Department of Mathematics \\ Federal Institute of Technology \\ ETH Zentrum \\ CH-8092 Zurich \\ Tel.: +4116326162 \\ Tel.: +4116321523 \\ mcneil@math.ethz.ch
}

